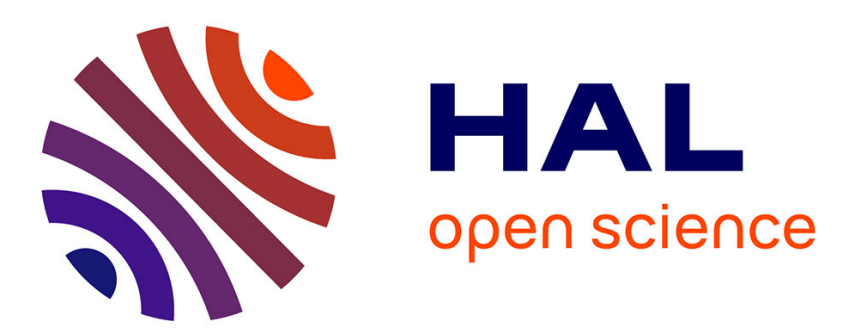

\title{
Observer-based control for linear systems with quantized output
}

\author{
Francesco Ferrante, Frédéric Gouaisbaut, Sophie Tarbouriech
}

\section{To cite this version:}

Francesco Ferrante, Frédéric Gouaisbaut, Sophie Tarbouriech. Observer-based control for linear systems with quantized output. European Control Conference 2014, Jun 2014, Strasbourg, France. pp.964-969. hal-00911570v2

\section{HAL Id: hal-00911570 \\ https://hal.science/hal-00911570v2}

Submitted on 2 Apr 2015

HAL is a multi-disciplinary open access archive for the deposit and dissemination of scientific research documents, whether they are published or not. The documents may come from teaching and research institutions in France or abroad, or from public or private research centers.
L'archive ouverte pluridisciplinaire HAL, est destinée au dépôt et à la diffusion de documents scientifiques de niveau recherche, publiés ou non, émanant des établissements d'enseignement et de recherche français ou étrangers, des laboratoires publics ou privés. 


\title{
Observer-based control for linear systems with quantized output*
}

\author{
Francesco Ferrante, Frédéric Gouaisbaut, Sophie Tarbouriech
}

\begin{abstract}
This paper is devoted to the observer-based control design for linear systems with quantized output. To this end, the output dynamic controller issued by the Luenberger observer is considered. Thus, through appropriate sector conditions which embed the quantization error, the global ultimate boundedness stability problem is tackled for the closed-loop system. Namely, to deal with the discontinuity induced by the quantizer, the whole treatise is carried out considering Krasovskii solutions to the closed-loop system. Furthermore, the controller is designed via a convex optimization setup, providing a constructive method that can be efficiently implemented.
\end{abstract}

\section{INTRODUCTION}

Nowadays, controlled systems are mainly heterogeneous systems interconnected by digital communication networks. The nature of these networks may often cause delays, asynchronism, and also quantization of the transmitted data (see [16] and references therein). In that sense, the old paradigm of an infinite precision of the available measures should be re-interpreted. Now, upstream from the network, some devices encode the data to be transmitted through the network and then re-encoded to be sent to the actuators. All these operations are no more transparent for the control and should be taken into account in the design [9]. The quantization phenomenon, which may also appears in some sensors used for position measurement of mechanical devices (optical incremental encoders), is a nonlinearity that transforms a signal with values in $\mathbb{R}$ into a signal with values in a discrete set. Introduced into a control loop, it often induces performance degradation, like limit cycles or chaotic phenomena [8] and they may even destabilize the closed-loop system. Thus in recent years, many studies have been dedicated to the analysis, control and observation of systems subject to quantization in order to reduce their impact on the closed loop. Several methods have been proposed in the literature depending on the nature of the quantization. When the quantized measures are without memory (also called uniform quantization), it is usually (at least for an unstable open loop) impossible to prove convergence to the origin. We can only show the convergence to an attractive and invariant set [24], [25]. One first method is to use the techniques issued from the robust control framework. The system is then modeled as a nominal system submitted to an uncertainty whose size is depending on the quantization precision. Quantization errors are then encapsulated into sector conditions and classical tools like small gain theorem or Lyapunov functions coupled with ISS properties [7] or S-procedure [1] are used to prove the ultimate boundedness of the states [11], [22]. These approaches have been extended to the $H_{\infty}$ control [18], $\mathcal{L}_{2}$ attenuation [21] and robust stabilization with respect to an input delay [10]. In the case of quantizer with memory (see [14] and references therein), as the precision of the quantizer may be infinite when approaching the origin, the stability properties of the closed loop can be obtained

The authors are with CNRS, LAAS, 7 Avenue Colonel du Roche, F-31400 Toulouse, France and Univ de Toulouse, UPS, ISAE, F-31400 Toulouse, France.

*This work has been supported by ANR project LimICoS contract number 12 BS03 00501.

ferrante, fgouaisb, tarbourelaas.fr using controls based on hybrid modeling [14], [4]. In that case, the quantization is scaled dynamically when approaching the origin. Note that in all these techniques, except in [15], it was assumed that all the states were measured which may be quite restrictive. In this paper, we focus on a linear system subject to output quantization and we aim at building an observer-based control. Following the methods of [15] and [2], we model the system as a nominal system subject to a quantization error, which is bounded. Afterwards, as shown in [11], this quantization error is described by a specific nonlinearity which satisfy some sector bound conditions. Then, by using a quadratic Lyapunov function for the overall system, we prove that the state converges to an ultimate bounded set surrounding the origin, for every initial condition. The obtained criterion is then expressed into an optimization procedure based on LMI. This procedure determines automatically the gain of the observer while reducing a measure of the size of the ultimate set. Furthermore, the proposed optimization setup can be also used to improve the closed-loop performance, when a quantized state-feedback is exploited to control the system. Although our work is related to the work proposed by Liberzon, the way we adopt to deal with the quantizer is different. Indeed in [15], for the quantizer an explicit definition is not provided, that is the quantizer is assumed to be a certain nonlinear map characterized by some properties, conversely in our paper the uniform quantizer is a well defined discontinuous nonlinear map and due to such a discontinuity we rely on proper concept of solution for discontinuous right-hand side differential equations. This allows even to deal with sliding motions on the quantization boundaries for the closed-loop system, which are often disregarded. Another difference with respect to the work of Liberzon is that we exhibit constructive results in the sense that the conditions arising for this paper can be efficiently implemented, thus the proposed approach represents a real synthesis tool. Concerning this latter aspect we can consider that, except for the saturation, our work represents an extension of the work in [22]. Moreover, it is worthwhile to point out that this paper provides a numerical procedure to deal with Krasovskii solutions and this represents a considerable novelty.

The paper is organized as follows. Section I presents the system under consideration and the problem we intend to solve. Section III is dedicated to the main theoretical conditions solution to the stated control problem. In Section IV, an algorithm procedure is proposed in order to efficiently implement the proposed theoretical conditions. In Section V, the effectiveness of the approach is illustrated through some examples. Finally, a conclusion ends the paper.

Notation: For every complex number $\omega, \mathfrak{R}(\omega)$ stands for the real part of $\omega . \mathbf{I}_{n}$ denotes the identity matrix whereas $\mathbf{1}_{n}$ denotes the vector of dimension $n$ with all components equal to 1 , furthermore $\mathbf{0}$ denotes the null matrix (equivalently the null vector) of appropriate dimensions. For a matrix $A \in \mathbb{R}^{n \times m}, A^{\prime}$ and $\operatorname{trace}(A)$ denote the transpose and the trace of $A$, respectively. $\operatorname{He}(A)=A+A^{\prime}$. For two symmetric matrices, $A$ and $B, A>B$ means that $A-B$ is positive definite. In partitioned symmetric matrices, the symbol $\star$ stands for symmetric blocks. The matrix $\operatorname{diag}\left\{A_{1} ; \ldots ; A_{n}\right\}$ is the 
block-diagonal matrix having $A_{1}, \ldots, A_{n}$ as diagonal blocks. For a vector $x \in \mathbb{R}^{n}, x_{(i)}$ denotes the $i-t h$ component and $x^{\prime}$ denotes the transpose of $x$, $|x|$ is the vector given by the absolute value of each component of $x$, whereas $\operatorname{sign}(x)$ is the componentwise signum function for which $\operatorname{sign}(0)=0$. The set $\Delta \mathbb{Z}^{p}$ is the set of the p-tuples of integers multiple of $\Delta$. The symbol $\langle\cdot, \cdot\rangle$ denotes the standard Euclidean inner product. For a set $U, \operatorname{Int}(U)$ denotes the interior of $U$. Let $A_{1} \ldots A_{m}$ be some given sets, $\times_{i=1}^{m} A_{i}=A_{1} \times A_{2} \times \ldots A_{m}$, where $\times$ stands for the standard Cartesian product.

\section{Problem Statement}

Consider the following continuous-time linear system with quantized output:

$$
\begin{aligned}
& \dot{x}=A x+B u \\
& y=\mathrm{q}(C x)
\end{aligned}
$$

where $x \in \mathbb{R}^{n}, u \in \mathbb{R}^{m}, y \in \mathbb{R}^{p}$ are respectively the state, the input and the measured output of the system. $A, B, C$ are constant matrices of appropriate dimensions. Whereas, the uniform quantizer is defined as follows:

$$
\mathrm{q}:\left\{\begin{array}{l}
\mathbb{R}^{p} \rightarrow \Delta \mathbb{Z}^{p} \\
z \mapsto \Delta \operatorname{sign}(z)\left\lfloor\frac{|z|}{\Delta}\right\rfloor
\end{array}\right.
$$

where $\Delta$ is an arbitrary strictly positive number which represents the quantization error bound i.e., $\left|\mathrm{q}\left(z_{i}\right)-z_{i}\right| \leqslant \Delta$ for all $i=1, \ldots, p$. Throughout the paper, we consider that system (1) is controlled through a dynamic output feedback controller of the form:

$$
\left\{\begin{array}{l}
\dot{\hat{x}}=A \hat{x}+B u+L(y-C \hat{x}) \\
u=K \hat{x}
\end{array}\right.
$$

where $\hat{x} \in \mathbb{R}^{n}, K$ and $L$ are constant matrices of appropriate dimensions to be designed.

Notice that the structure of the system (3) is based on a Luenberger observer, and thus the state $\hat{x} \in \mathbb{R}^{n}$ can be viewed as an estimate of the state $x$. Hence, the closed-loop system (1)-(3) can be written as

$$
\left\{\begin{array}{l}
\dot{x}=A x+B K \hat{x} \\
\dot{\hat{x}}=(A+B K-L C) \hat{x}+L \mathrm{q}(C x)
\end{array}\right.
$$

Classically by defining the error $\varepsilon=x-\hat{x}$ and the nonlinearity $\Psi(v)=\mathrm{q}(v)-v$, the closed-loop system (1)-(3) reads:

$$
\left\{\begin{array}{l}
\dot{x}=(A+B K) x-B K \varepsilon \\
\dot{\varepsilon}=(A-L C) \varepsilon-L \Psi(C x) .
\end{array}\right.
$$

Note that system (5), due the presence of the nonlinearity $\Psi(\cdot)$, is described by a discontinuous right-hand side differential equation. For this, as pointed out in [4], Carathéodory solutions to system (5) may not exist, thus relying on a suitable concept of solution is needed. Especially in this paper we focus on Krasovskii solutions to system (5), the existence of such solutions is guaranteed under very mild conditions, which are naturally satisfied by the function $\Psi(\cdot)$, for an exhaustive treatise about these aspects, we invite to see [6]. It seems to be suitable to recall that Krasovskii solutions of (5) are Carathéodory solutions of the following differential inclusion

$$
\left[\begin{array}{c}
\dot{x} \\
\dot{\varepsilon}
\end{array}\right] \in \underbrace{\left[\begin{array}{c}
(A+B K) x-B K \varepsilon \\
(A-L C) \varepsilon-L \mathscr{K}(\Psi(C x))
\end{array}\right]}_{\mathscr{W}(x, \varepsilon)}
$$

where the Krasovskii operator $\mathscr{K}(f(x))$ is defined as

$$
\mathscr{K}(f(x))=\bigcap_{\delta>0} \overline{\operatorname{co}}(f(\mathbb{B}(x, \delta))) .
$$

For further details see [3] and [4]. Especially, in this case for $i=1 \ldots p$, the above mentioned Krasovskii operator, by continuity arguments, applied to the $i$-th component of the nonlinearity $\Psi(C x)$ provides the following inclusion:

$\mathscr{K}\left(\Psi_{(i)}\left(C_{(i)} x\right)\right) \subseteq\left\{\begin{array}{l}\Psi_{(i)}\left(C_{(i)} x\right) \text { if } C_{(i)} x \neq \Delta \mathbb{Z} \backslash\{0\}, \\ \left\{\lambda \operatorname{sign}\left(C_{(i)} x\right) \Delta, \lambda \in[-1,0]\right\} \text { otherwise, }\end{array}\right.$

Figure 1 depicts the right-hand side of the above inclusion in the scalar case. Notice also that, as shown in [17] Theorem

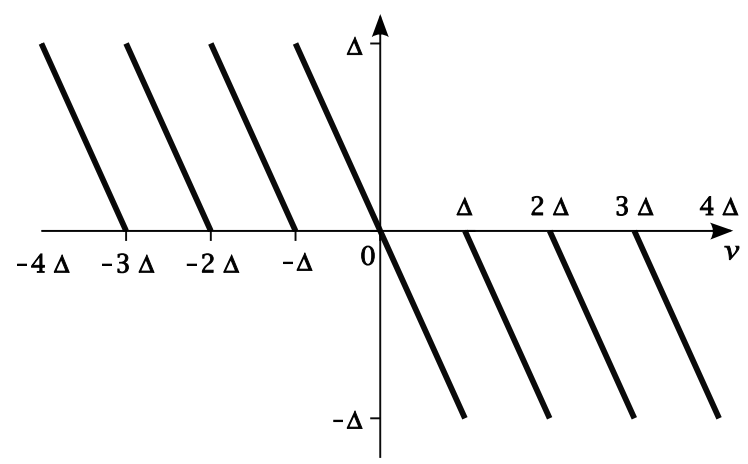

(a). The function $\Psi(v)$

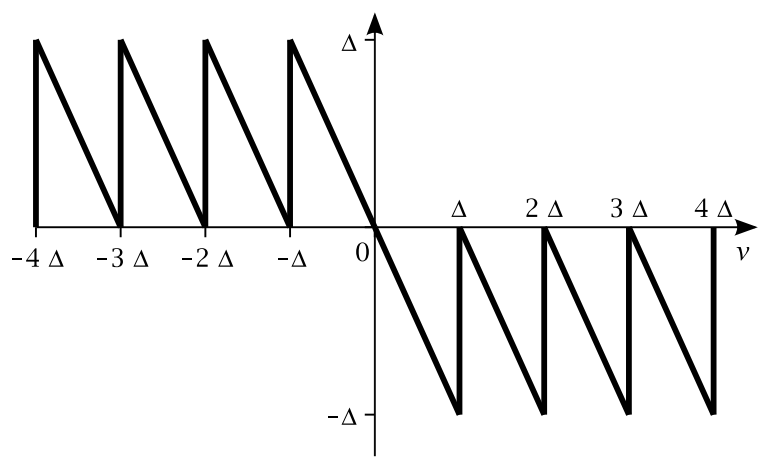

(b). The set-valued map $\mathscr{K}(\Psi(v))$

Fig. 1. Comparison between the function $\Psi(\cdot)$ and the associated set-valued map $\mathscr{K}(\Psi(v))$

1 , for every $v \in \mathbb{R}^{p}$, one has

$$
\mathscr{K}(\Psi(v)) \subseteq \underset{i=1}{p} \mathscr{K}\left(\Psi\left(v_{(i)}\right)\right) .
$$

Moreover, it should be noticed that due to the dead-zone effect induced by the quantizer, achieving the asymptotic stability property of the origin for closed-loop system (4) (and then equivalently for system (5)), with respect to Krasovskii solution, is not possible if the matrix $A$ is not Hurwitz. Indeed, there exists a neighborhood $\mathcal{B}$ of the origin such that $\mathrm{q}(C x)=0, \forall x \in \mathcal{B}$ then system (4) behaves locally as the linear system described by:

$$
\left[\begin{array}{c}
\dot{x} \\
\dot{\hat{x}}
\end{array}\right]=\left[\begin{array}{cc}
A & B K \\
0 & A+B K-L C
\end{array}\right]\left[\begin{array}{l}
x \\
\hat{x}
\end{array}\right]
$$


which is unstable if $A$ is not Hurwitz. Nevertheless, assuming $A$ to be Hurwitz is too much restrictive and we aim at establishing a practical stability property for the closed-loop system. To this end, we rely on the ultimate boundedness property proposed in [13]. Hence, the problem we intend to solve is summarized as follows:

Problem 1 (Practical stabilization): Determine the gains $K$ and $L$ and characterize a set $\mathcal{S}_{u} \subset \mathbb{R}^{2 n}$ such that for every initial condition $\left[x^{\prime}(0) \varepsilon^{\prime}(0)\right]^{\prime} \in \mathbb{R}^{2 n}$ the resulting Krasovskii solutions to closed-loop system (5) are ultimately bounded in $S_{u}$.

\section{MAin Result}

In order to solve Problem 1, we firstly show some sector conditions for $\Psi(\cdot)$, which will be useful in the sequel. Concerning the function $\Psi(v)$ defined in (2) the following Lemma holds:

Lemma 1: The function $\Psi(v)$ satisfies the following conditions:

$$
\begin{aligned}
& \Psi^{\prime}(v) S_{1} \Psi(v)-\Delta^{2} \mathbf{1}_{p}^{\prime} S_{1} \mathbf{1}_{p} \leqslant 0, \forall v \in \mathbb{R}^{p} \\
& \Psi^{\prime}(v) S_{2}(\Psi(v)+v) \leqslant 0, \forall v \in \mathbb{R}^{p}
\end{aligned}
$$

for any diagonal positive definite matrices $S_{1}, S_{2} \in \mathbb{R}^{p \times p}$.

Proof: According to the definition of $\Psi(v)$ one gets:

$$
\Psi_{i}^{2}\left(v_{(i)}\right)-\Delta^{2} \leqslant 0
$$

then taking $p$ positive arbitrarily scalars $s_{1}^{1}, \ldots, s_{p}^{1}$ it follows that $s_{i}^{1} \Psi_{i}^{2}\left(v_{(i)}\right)-\Delta^{2} s_{i}^{1} \leqslant 0$ for every $i=1, \ldots, p$. Hence by summing for $i=1, \ldots, p$, by defining $S_{1}=\operatorname{diag}\left\{s_{i}^{1} ; \ldots ; s_{p}^{1}\right\}$, from the foregoing relation, one gets (10). Concerning relation (11), take $p$ positive arbitrarily scalars $s_{1}^{2}, \ldots, s_{p}^{2}$ and set $S_{2}=\operatorname{diag}\left\{s_{i}^{2} ; \ldots ; s_{p}^{2}\right\}$. Then, by noticing that for every $v$ belonging to $\mathbb{R}^{p}, \Psi_{(i)}\left(v_{i}\right)\left(v_{i}+\Psi_{(i)}\left(v_{i}\right)\right) \leqslant 0$, for $i=1, \ldots, p$, by similar arguments as in the former case, it follows that (11) holds.

Remark 1: Notice that, even if the sector conditions provided by the foregoing Lemma concern the single-valued map $\Psi(\cdot)$, it turns out that such conditions, as pointed out by Figure 1, can be exploited to deal with the set-valued map $\mathscr{K}(\Psi(v))$ as well. Indeed, it can be easily shown that for every $v \in \mathbb{R}^{p}$ and for every $i \in\{1, \ldots, p\}$, every element of the set $\times_{i=1}^{p} \mathscr{K}\left(\Psi(v)_{(i)}\right)$ satisfies inequalities (10) and (11) and due to the inclusion reported in (8), it follows that every element of the set $\mathscr{K}(\Psi(v))$ satisfies (10) and (11) as well.

Now, considering closed-loop system (5), the following result provides a solution to Problem 1.

Proposition 1: If there exist two symmetric positive definite matrices $P_{1}, P_{2} \in \mathbb{R}^{n \times n}$, two diagonal positive definite matrices $S_{1}, S_{2} \in \mathbb{R}^{p \times p}$, two matrices $K \in \mathbb{R}^{m \times n}$ and $L \in \mathbb{R}^{n \times p}$ and a positive scalar $\tau$ such that:

$$
M_{1}<\mathbf{0}
$$

where $M_{1}$ is defined in (15) (at the top of next page), then $K, L$ and

$$
\mathcal{S}_{u}=\left\{\left[x^{\prime} \varepsilon^{\prime}\right]^{\prime} \in \mathbb{R}^{2 n}: x^{\prime} P_{1} x+\varepsilon^{\prime} P_{2} \varepsilon \leqslant 1\right\}
$$

are solution to Problem 1.

Proof: Consider the following quadratic Lyapunov function

$$
V(x, \varepsilon)=x^{\prime} P_{1} x+\varepsilon^{\prime} P_{2} \varepsilon
$$

with $P_{1}=P_{1}^{\prime}>\mathbf{0}$ and $P_{2}=P_{2}^{\prime}>\mathbf{0}$. Define

$$
\dot{V}(x, \varepsilon)=\langle\nabla V(x, \varepsilon), f\rangle, \forall f \in \mathscr{W}(x, \varepsilon)
$$

where $\mathscr{W}(x, \varepsilon)$ is defined in (6). We want to prove that there exist two positive scalars $\bar{\beta}_{1}$ and $\bar{\beta}_{2}$ such that

$$
\dot{V}(x, \varepsilon) \leqslant-\bar{\beta}_{1} x^{\prime} x-\bar{\beta}_{2} \varepsilon^{\prime} \varepsilon, \forall\left[x^{\prime} \varepsilon^{\prime}\right]^{\prime} \in \mathbb{R}^{2 n} \backslash \operatorname{Int}\left(\mathcal{S}_{u}\right) .
$$

where the set $\mathcal{S}_{u}$ is defined in (14). By using S-procedure argument in order to prove relation (16) it suffices to prove that there exists a positive scalar $\tau$ such that for every $[x, \varepsilon] \in$ $\mathbb{R}^{2 n}$ and for every $f \in \mathscr{W}(x, \varepsilon)$

$$
\dot{V}(x, \varepsilon)-\tau\left(1-x^{\prime} P_{1} x-\varepsilon^{\prime} P_{2} \varepsilon\right) \leqslant-\bar{\beta}_{1} x^{\prime} x-\bar{\beta}_{2} \varepsilon^{\prime} \varepsilon .
$$

To this end, notice that:

$$
\begin{aligned}
& \dot{V}(x, \varepsilon)=x^{\prime}\left((A+B K)^{\prime} P_{1}+P_{1}(A+B K)\right) x+ \\
& +\varepsilon^{\prime}\left((A-L C)^{\prime} P_{2}+P_{2}(A-L C)\right) \varepsilon+ \\
& -2 x^{\prime} P_{1} B K \varepsilon-2 \varepsilon^{\prime} P_{2} L w, \forall w \in \mathscr{K}(x, \varepsilon) .
\end{aligned}
$$

Moreover, since for every $w \in \mathscr{K}(x, \varepsilon)$ relations (10) and (11) are satisfied, then once again according to S-procedure arguments, to show relation (16) it suffices to show that there exist some diagonal positive definite matrices $S_{1}, S_{2}$ and a positive scalar $\tau$ such that:

$$
\begin{aligned}
& \dot{V}(x, \varepsilon)-2 w^{\prime} S_{2} C x-2 w^{\prime} S_{2} w-w^{\prime} S_{1} w+\mathbf{1}_{p}^{\prime} S_{1} \mathbf{1}_{p} \Delta^{2} \\
& -\tau\left(1-x^{\prime} P_{1} x-\varepsilon^{\prime} P_{2} \varepsilon\right) \leqslant-\bar{\beta}_{1} x^{\prime} x-\bar{\beta}_{2} \varepsilon^{\prime} \varepsilon \forall[x, \varepsilon] \in \mathbb{R}^{2 n}
\end{aligned}
$$

Now, defining $\xi=\left[\begin{array}{llll}x^{\prime} & \varepsilon^{\prime} & w^{\prime} & \mathbf{1}_{p}^{\prime}\end{array}\right]^{\prime}$ the right hand side of relation (19) can be re-written equivalently as follows:

$$
\xi^{\prime} M_{1} \xi
$$

where $M_{1}$ is defined in (15). Therefore, the satisfaction of relation (13) implies that there exist four positive real scalars $\beta_{1}, \beta_{2}, \beta_{3}, \beta_{4}$ such that

$$
\xi^{\prime} M_{1} \xi \leqslant-\xi^{\prime} \operatorname{diag}\left\{\beta_{1} \mathbf{I}_{n} ; \beta_{2} \mathbf{I}_{n} ; \beta_{3} \mathbf{I}_{p} ; \beta_{4} \mathbf{I}_{p}\right\} \xi .
$$

Then, by the virtue of definition of $\xi$, one has

$$
\xi^{\prime} M_{1} \xi \leqslant-\bar{\beta}_{1} x^{\prime} x-\bar{\beta}_{2} \varepsilon^{\prime} \varepsilon
$$

hence relation (19) holds implying that inequality (17) is satisfied as well as inequality (16). Thus according to [3] and [13], it follows that the Krasovskii solutions to (5) are ultimately bounded in the set $\mathcal{S}_{u}$ defined in (14) which concludes the proof.

Classically, it appears in Proposition 1 that the gains $K$ and $L$ cannot be computed simultaneously through a convex problem [20]. However, an adequate change of variable allows us to compute the gain $L$ as described below.

Corollary 1: If there exist two symmetric positive definite matrices $P_{1}, P_{2} \in \mathbb{R}^{n \times n}$, two diagonal positive definite matrices $S_{1}, S_{2} \in \mathbb{R}^{p \times p}$, two matrices $K \in \mathbb{R}^{m \times n}$ and $J \in \mathbb{R}^{n \times p}$ and a positive scalar $\tau$ such that:

$$
M_{2}<\mathbf{0}
$$

with $M_{2}$ defined in (24) (at the top of next page), then $K$, $L=P_{2}^{-1} J$ and $\mathcal{S}_{u}$ defined in (14) are solution to Problem 1 . Proof: Setting $P_{2} L=J$ in (15), the result immediately follows from Proposition 1. 


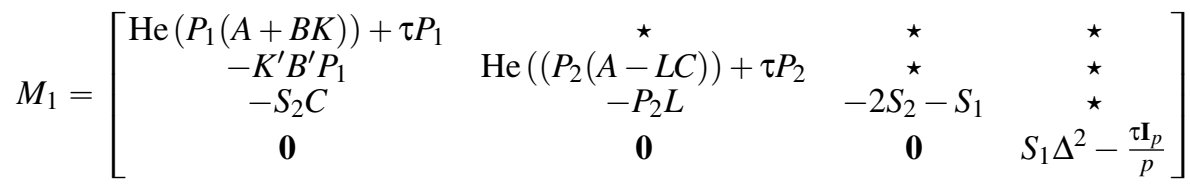

$$
\begin{aligned}
& M_{2}=\left[\begin{array}{cccc}
\operatorname{He}\left(P_{1}(A+B K)\right)+\tau P_{1} & \star & \star & \star \\
-K^{\prime} B^{\prime} P_{1} & \operatorname{He}\left(P_{2} A-J C\right)+\tau P_{2} & \star & \star \\
-S_{2} C & -J & -2 S_{2}-S_{1} & { }^{\star} \\
\mathbf{0} & \mathbf{0} & \mathbf{0} & S_{1} \Delta^{2}-\frac{\tau \mathbf{I}_{p}}{p}
\end{array}\right]
\end{aligned}
$$

\section{Computational Issues}

Relation (23) becomes linear with respect to the decision variables if $\tau$ and $K$ are fixed. In this case relation (23) is linear in $P_{1}, P_{2}, S_{1}, S_{2}, S_{3}$ and $J$.

The selection of $\tau$ is not a difficult task and it can be selected through an iterative search. In our proposal, $K$ is supposed to be known and it allows us to compare our solution with those obtained in the static state feedback case, $u=K \mathrm{q}(x)$, which is treated in [22]. In fact, thanks to the results shown in [22] and also in [10], one obtains a stabilizing gain $K$ and a set $S_{u}^{K} \subset \mathbb{R}^{n}$ in which the resulting closed-loop trajectories are ultimately bounded.

One idea is to compute the gain $L$ (for Proposition 1 or Corollary 1) aiming at reduce the size of the projection in the $x$-coordinates of the set $S_{u}$ defined in (14). Another idea is computing the gain $L$ in order to reduce the chattering behavior observed on the time evolution of the state and/or the input of the closed-loop system, due to the presence of the quantizer. In that case, the chattering phenomenon is reduced by the filtering effect of the observer [23]. These two aspects will be illustrated in the numerical examples.

Moreover, the conditions of Proposition 1 or Corollary 1 can be used in a convex optimization setup allowing to minimize the size of the set $S_{u}$ defined in (14). Several optimality criteria can be considered, depending on the size criterion chosen for measuring the set $\mathcal{S}_{u}$.

For example, as shown in [1], volume, minor axis and direction of interests could be a good measure of an ellipsoidal set. Among the various criteria provided by the literature, we adopt a trace maximization criterion, in order to induce, through the optimization procedure, an uniform contraction on $\mathcal{S}_{u}$. Furthermore, it is important to note that we are more specifically interested by minimizing the size of $\mathcal{S}_{u}$ in the $x$ direction. Hence, we consider the maximization of the trace of $P_{1}$. An optimization of the set $\mathcal{S}_{u}$ can be achieved through the following algorithm:

Algorithm 1:

- Step 1 Initializing $K$ and $\tau$. For example $K$ can be fixed at the value issued from the static state feedback problem.

- Step 2 Given $K$ and $\tau$ solve the following LMI optimization problem:

$$
\begin{aligned}
& \min _{P_{1}, P_{2} S_{1}, S_{2}, J}-\operatorname{trace}\left(P_{1}\right) \\
& \text { subject to }(23), P_{1}>\mathbf{0}, P_{2}>\mathbf{0}, S_{1}>\mathbf{0} ; \\
& S_{2}>\mathbf{0} ;
\end{aligned}
$$

which gives $L=P_{2}^{-1} J$.
- Step 3 Given $K, L$ and $\tau$ solve the following LMI optimization problem:

$$
\begin{aligned}
& \min _{P_{1}, P_{2} S_{1}, S_{2}}-\operatorname{trace}\left(P_{1}\right) \\
& \text { subject to }(13), P_{1}>\mathbf{0}, P_{2}>\mathbf{0}, S_{1}>\mathbf{0} ; \\
& S_{2}>\mathbf{0} ;
\end{aligned}
$$

The Step 3 is performed in order to shrink as much as possible the ultimate set $S_{u}$.

Remark 2: We could add in (25) and (26) a constraint imposing that the projection along the $x$-direction of the set $S_{u}$ defined in (14) is included in the set $S_{u}^{K}$ resulting of the static state feedback case. To do this, supposing that $S_{u}^{K}$ is defined from a symmetric positive definite matrix $P_{K}$ as $\mathcal{S}_{u}^{K}=\left\{x \in \mathbb{R}^{n}: x^{\prime} P_{K} x \leqslant 1\right\}$, it suffices to add the constraint $P_{1}-P_{K} \geqslant \mathbf{0}$, being $P_{K}$ given ${ }^{1}$.

\section{NUMERICAL EXAMPLES}

Example 1: Consider the system (1) defined by the following data:

$$
\begin{array}{ll}
A=\left[\begin{array}{cc}
0 & 1 \\
1 & -1
\end{array}\right], & B=\left[\begin{array}{l}
1 \\
1
\end{array}\right] \\
C & =\left[\begin{array}{ll}
1 & 0
\end{array}\right], \quad \Delta=0.25 .
\end{array}
$$

By the conditions proposed in [22] one gets $K=$ $\left[\begin{array}{ll}-1.7047-1.0566\end{array}\right]$ then by fixing $\tau=0.8$ the optimization scheme proposed in (25) leads to $L^{\prime}=\left[\begin{array}{lll}10.618 & 6.5623\end{array}\right]$.

In Figure 2, several closed-loop trajectories converging in a finite time in $S_{u}$ are plotted. As expected, the optimization procedure leads to a small set $\mathcal{S}_{u}$, which represents to a good estimation of the real ultimate set in which the closed-loop system trajectories are captured.

Example 2 (Furuta pendulum [12]): We consider a Furuta pendulum designed by Quanser [12]. In that case the whole state vector is measured and exploited to control the system. However, such a measure is performed by a device that, through a digital post-processing of the data delivered by an optical incremental encoder [19], provides a finite resolution measure of the state. Such a phenomenon can be modeled by a uniform quantization process characterized by $\Delta=2 \pi / 1024 \approx 0.006$. Specifically, the plant is controlled by static state feedback by means of the quantized measured state, that is:

$$
u=K \mathrm{q}(x)
$$

\footnotetext{
${ }^{1}$ In order to prevent the occurrence of a too large value for the gain $L$ (high observer gain), one can add in (25) an additional constraint, specifying a certain pole placement for the eigenvalues of the matrix $A-L C$. For example, one can impose that the eigenvalues of the matrix $A-L C$, denoted by $\lambda_{i}$, belong to a strip of the complex plane as $-\alpha \leqslant \Re\left(\lambda_{i}\right)<0, \alpha>0$ [5].
} 


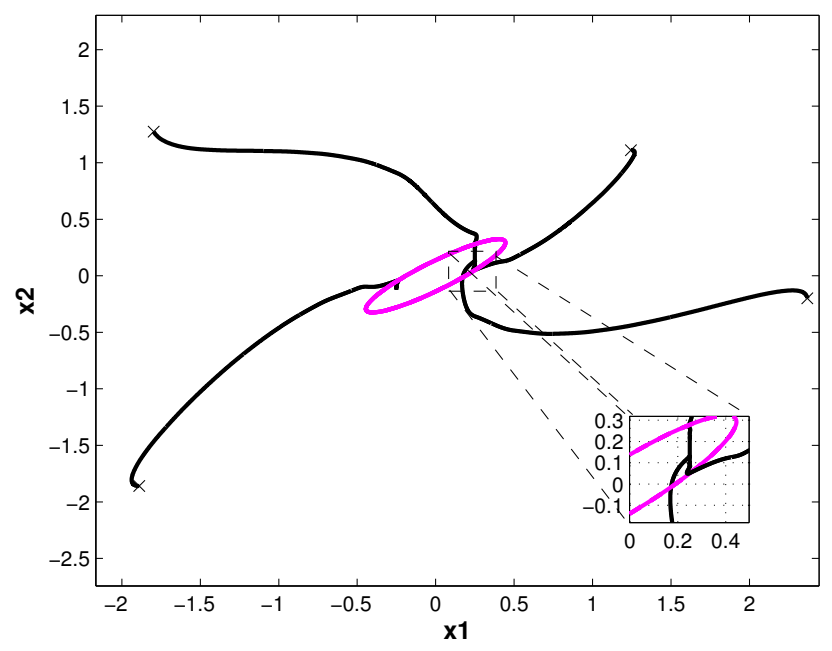

Fig. 2. Projection of the set $\mathcal{S}_{u}$ on the $\left(x_{1}, x_{2}\right)$ plane (magenta) and plant convergent trajectories obtained setting $\hat{x}(0)=0$.

where:

$$
K=\left[\begin{array}{llll}
1 & -24.9103 & 2.0751 & -3.6549
\end{array}\right] \text {. }
$$

The considered plant model is nonlinear but it has been linearized around the unstable equilibrium point, providing the following linear model:

$$
\dot{x}=\left[\begin{array}{cccc}
0 & 0 & 1 & 0 \\
0 & 0 & 0 & 1 \\
0 & 39.32 & -14.52 & 0 \\
0 & 81.78 & -13.98 & 0
\end{array}\right] x+\left[\begin{array}{c}
0 \\
0 \\
25.54 \\
24.59
\end{array}\right] u
$$

where $x_{1}, x_{2}$ represent respectively the base angle and the pendulum angle, whereas $x_{3}=\dot{x}_{1}, x_{4}=\dot{x}_{2}$. Furthermore in the studied case, we assume that the control system designer has not access to the gain $K$, which cannot be modified ${ }^{2}$. Thus in this situation one can attempt to improve the closedloop performances, exploiting the filtering action provided by controller (3) on the measured variables. To this end, we want to test our methodology considering $C=\mathbf{I}_{n}$ and successively comparing it to static state feedback controller (28), which is analyzed by using conditions stated in [22]. Therefore, by fixing:

$$
\tau=1.3
$$

the proposed optimization procedure (25) provides the following observer gain:

$$
L=\left[\begin{array}{cccc}
2.2351 & -3.3288 & -0.0365 & -13.4360 \\
-0.1233 & 1.8923 & 0.0186 & 7.2954 \\
-0.2198 & 3.3726 & 0.0332 & 13.0029 \\
-0.9298 & 14.2687 & 0.1405 & 55.0124
\end{array}\right]
$$

The projections of the set $\mathcal{S}_{u}$ on the $\left(x_{i}, x_{j}\right)$ plane are reported in Figure 3. Clearly, designing an observer allow us to reduce the estimation of the ultimate set compared with the static state feedback case. Furthermore, Figure 4 and Figure 5 depict respectively the time-evolution of the

\footnotetext{
${ }^{2}$ Notice that such situations are typical in control engineering practice, due to the presence of technological constraints. Hence analyzing this example is undoubtedly interesting. Namely, in the present case, the gain $K$ results from an LQ control setup performed on linear model (29) neglecting the quantization effect.
}
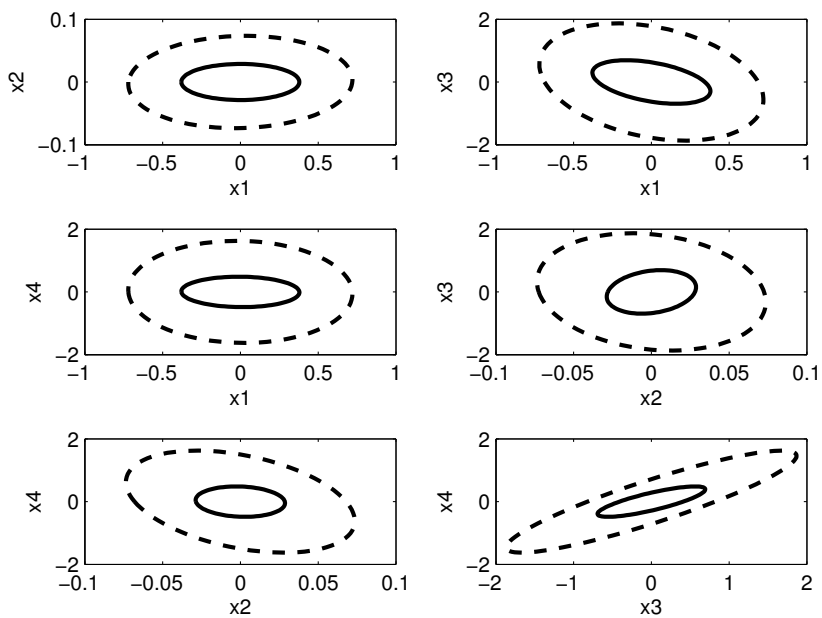

Fig. 3. Projections of the set $\mathcal{S}_{u}$ on the $\left(x_{i}, x_{j}\right)$ planes $i, j \in\{1,2,3,4\}, i \neq j$ : static controller (dashed) and observer-based controller (plain)

state and the control values resulting from the static state feedback controller (see Figure 4.a and Figure 5.a) and from the observer-based controller (see Figure 4.b and Figure 5.b). One can then observe that, effectively thanks to the filtering action of the controller (3), the high frequency oscillations affecting the state behavior is reduced in terms of magnitude as well as the chattering phenomenon affecting the control variable in the static state feedback case is alleviated (see Figure 5). That highlights the real benefits provided in using an observer-based control, even if whole the state is measurable, confirming our guess.

\section{CONCLUSION}

This paper proposed a methodology to design an observerbased control for a linear system with quantized output, in order to achieve the global ultimate boundedness stabilization property of the closed-loop system with respect to Krasovskii solutions. Furthermore, the observer design is performed computing the observer gain by a convex optimization setup, allowing to reduce the size of the ultimate set. Moreover, numerical solutions have shown that, even if a quantized measure of the state is available, adopting an observer-based control can improve the closed-loop system performance. This paper offers several sparks that could be studied in future works. Among these, the design of a fullorder dynamic filter, with a different structure from that one proposed in (3), should be addressed. Moreover, inspired by the work of [22], one could analyze the observer-based control design problem, for linear systems whose output results in a sandwich between saturation and quantization nonlinearity. Another open question consists of designing also the gain $K$ by a convex optimization setup.

\section{REFERENCES}

[1] S. Boyd, L. E. Ghaoui, E. Feron, and V. Balakrishnan. Linear Matrix Inequalities in System and Control Theory. Society for Industrial and Applied Mathematics, June 1997.

[2] R. W. Brockett and D. Liberzon. Quantized feedback stabilization of linear systems. IEEE Transactions on Automatic Control, 45(7):12791289,2000

[3] F. Ceragioli and C. De Persis. Discontinuous stabilization of nonlinear systems: Quantized and switching controls. Systems \& control letters, 56(7):461-473, 2007.

[4] F. Ceragioli, C. De Persis, and P. Frasca. Discontinuities and hysteresis in quantized average consensus. Automatica, 47:1919-1928, 2011. 


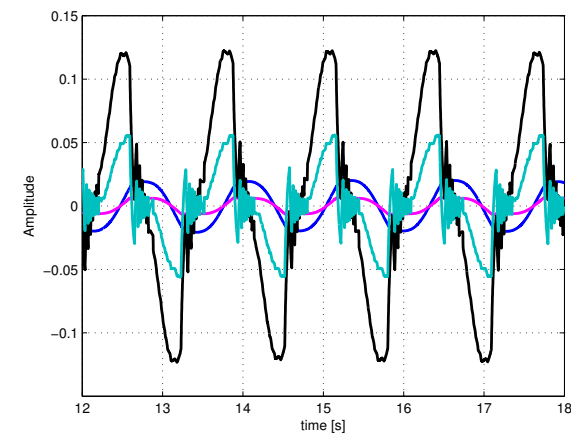

(a) Static state feedback controller

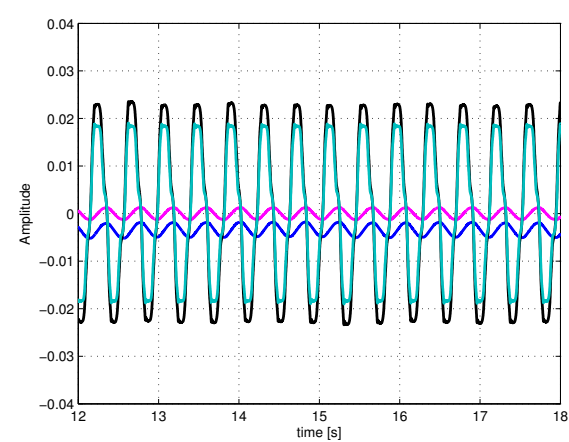

(b) Observer-based controller

Fig. 4. Closed-loop steady-state behavior, concerning the plant state $x$, arising from $x(0)=\left[\begin{array}{llll}0 & 0.1 & 0 & 0\end{array}\right]^{\prime}, \hat{x}(0)=0 . x_{1}(t)$ (blue), $x_{2}(t)$ (magenta), $x_{3}(t)$ (black), $x_{4}(t)$ (light-blue)

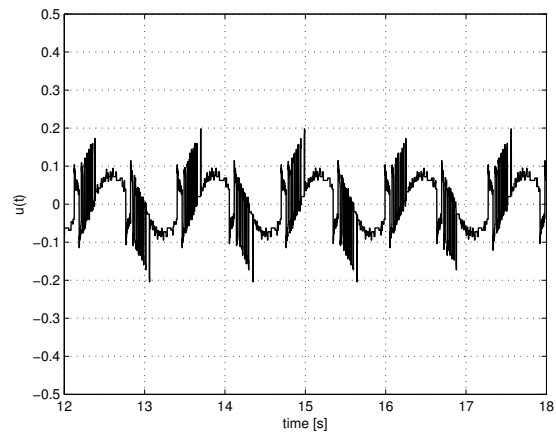

(a) Static state feedback controller

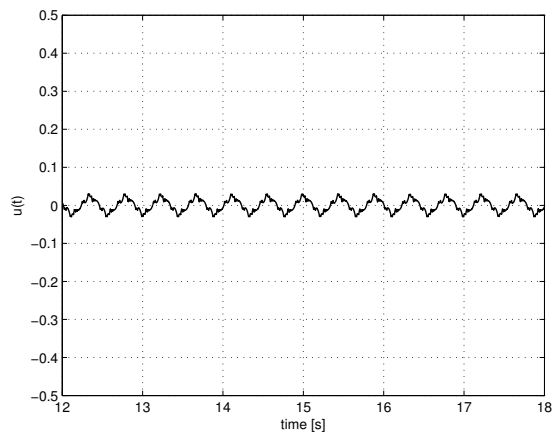

(b) Observer-based controller

Fig. 5. Control values in both cases related to simulation shown in Figure 4
[5] M. Chilali, P. Gahinet, and P. Apkarian. Robust pole placement in LMI regions. IEEE Transactions on Automatic Control, 44(12):2257-2270, 1999.

[6] J. Cortès. Discontinuous dynamical systems - a tutorial on solutions, nonsmooth analysis, and stability. IEEE Control Systems Magazine, 28(3):36-73, 2008

[7] D. F. Coutinho, M. Fu, and C. E. de Souza. Input and output quantized feedback linear systems. IEEE Transactions on Automatic Control, 55(3):761 -766, march 2010 .

[8] D. F. Delchamps. Stabilizing a linear system with quantized state feedback. IEEE Transactions on Automatic Control, 35(8):916 -924, aug 1990.

[9] N. Elia and S.K. Mitter. Stabilization of linear systems with limited information. IEEE Transactions on Automatic Control, 46(9):1384 1400, September 2001.

[10] E. Fridman and M. Dambrine. Control under quantization, saturation and delay: An LMI approach. Automatica, 45:2258-2264, 2009.

[11] M. Fu and L. Xie. The sector bound approach to quantized feedback control. IEEE Transactions on Automatic Control, 50(11):1698-1711, 2005 .

[12] Quanser Consulting Inc. Rotary Inverted Pendulum ROTPEN, 1998.

[13] H. K. Khalil. Nonlinear Systems. Prentice-Hall, 3rd edition, 2002.

[14] D. Liberzon. Switching in Systems and Control. Birkhauser, Boston, USA, 2003.

[15] D. Liberzon. Observer-based quantized output feedback control of nonlinear systems. Control Automation, 2007. MED '07. Mediterranean Conference on, pages 1-5, june 2007.

[16] I. Lopez Hurtado, C. T. Abdallah, and C. Canudas-de Wit. Contro under limited information: Special issue (part i). International Journal of Robust and Nonlinear Control, 19(16):1767-1769, 2009.

[17] B. Paden and S. Sastry. A calculus for computing Filippov's differential inclusion with application to the variable structure contro of robot manipulators. IEEE Transactions on Circuits and Systems, 34(1), 1987.

[18] B. Picasso and P. Colaneri. Stabilization of discrete-time quantized linear systems: An $H_{\infty} / l_{1}$ approach. In 47th IEEE conference on decision and control, pages 2868-2873, Cancun, Mexico, December 2008

[19] P. Ripka and A. Tipek. Modern sensors handbook. John Wiley \& Sons, 2013.

[20] S. Tarbouriech, G. Garcia, J.M. Gomes da Silva Jr. and I. Queinnec. Stability and stabilization of linear systems with saturating actuators. Springer, 2011.

[21] S. Tarbouriech and F. Gouaisbaut. L2 stability for quantized linear systems with saturations. In World IFAC Congress (IFAC 2011), pages 2338-2343, Milano (Italy), 2011.

[22] S. Tarbouriech and F. Gouaisbaut. Control design for quantized linear systems with saturations. IEEE Transactions on Automatic Control, 57(7): $1883-1889$, july 2012.

[23] V. I. Utkin. Sliding modes in control and optimization, volume 116 Springer-Verlag Berlin, 1992.

[24] W. S. Wong and R. W. Brockett. Systems with finite communication bandwidth constraints. i. state estimation problems. IEEE Transactions on Automatic Control, 42(9):1294-1299, September 1997.

[25] W. S. Wong and R.W. Brockett. Systems with finite communication bandwidth constraints. ii. stabilization with limited information feedback. IEEE Transactions on Automatic Control, 44(5):1049-1053, May 1999. 